This is an Accepted Manuscript of an article published by Taylor \& Francis in Journal of Interprofessional Care on 30 March 2016, available online:

http://www.tandfonline.com/10.3109/13561820.2015.1122582.

\title{
A Design Thinking Approach to Evaluating Interprofessional Education
}

Peter Cahn; Andrew Bzowyckyj; Lauren Collins; Alan Dow; Kristen Goodell; Alex Johnson; David Klocko; Mary Knab; Kathryn Parker; Scott Reeves; Brenda Zierler

\section{Abstract}

The complex challenge of evaluating the impact of interprofessional education (IPE) on patient and community health outcomes is well documented. Recently, at the Radcliffe Institute for Advanced Study in the United States, leaders in health professions education met to help generate a direction for future IPE evaluation research. Participants followed the stages of design thinking, a process for human-centered problem solving, to reach consensus on recommendations. The group concluded that future studies should focus on measuring an intermediate step between learning activities and patient outcomes. Specifically, knowing how IPE-prepared students and preceptors influence the organizational culture of a clinical site as well as how the culture of clinical sites influence learners' attitudes about collaborative practice will demonstrate the value of educational interventions. With a mixed methods approach and an appreciation for context, researchers will be able to identify the factors that foster effective collaborative practice and, by extension, promote patient-centered care.

Keywords: interprofessional education, interprofessional collaborative practice, program evaluation, design thinking, organizational culture 


\section{Introduction}

As many health professions have moved to include interprofessional education (IPE) in accreditation standards and many universities have developed IPE learning activities, answering whether the benefits of IPE outweigh the costs has become more urgent (Brandt, Lutfiyya, King, \& Chioreso, 2014). In April 2015, an Institute of Medicine committee released recommendations on how best to measure the long-term impact of IPE on patient and population health (IOM, 2015). The authors propose a conceptual model as a way to standardize research around commonly accepted markers along the learning continuum. This theory-driven approach to evaluation has been successfully implemented in medical education to answer not just what program outcomes are but how and why they occurred (Parker, Burrows, Nash, \& Rosenblum, 2011).

A month before the IOM report appeared, twelve health professions educators and researchers from the United States, Canada, and the United Kingdom convened for a two-day seminar to generate IPE evaluation strategies. Although we did not use the report as a basis for discussion, the conclusions we reached contribute to the development of a widely applicable research agenda that measures the relationship between IPE and collaborative practice by identifying intermediate steps on the path between education and practice. Specifically, we conceptualized the potential of IPE interventions to affect organizational culture at a practice site that will promote collaborative care. In turn, more effective collaborative practice will lead to improved health outcomes. 


\section{Methods}

The seminar structure followed the generative process of design thinking. Popularized by the Silicon Valley consulting firm IDEO, design thinking spurs creative solutions to problems through collaboration and experimentation (Brown, 2008). By following five sequential stages, design thinkers create and test potential solutions to a central challenge (Figure 1). When used by technology companies to test users' experiences of software, the process can take months and require extensive engineering and fieldwork. The stages, however, are flexible enough to adapt to different time scales and industries (Zuber, Alterescu, \& Chow, 2005). To fit the framework of an academic seminar, we condensed the timeline to two days and substituted conceptual models for actual product prototypes while remaining faithful to the structured process for innovation.

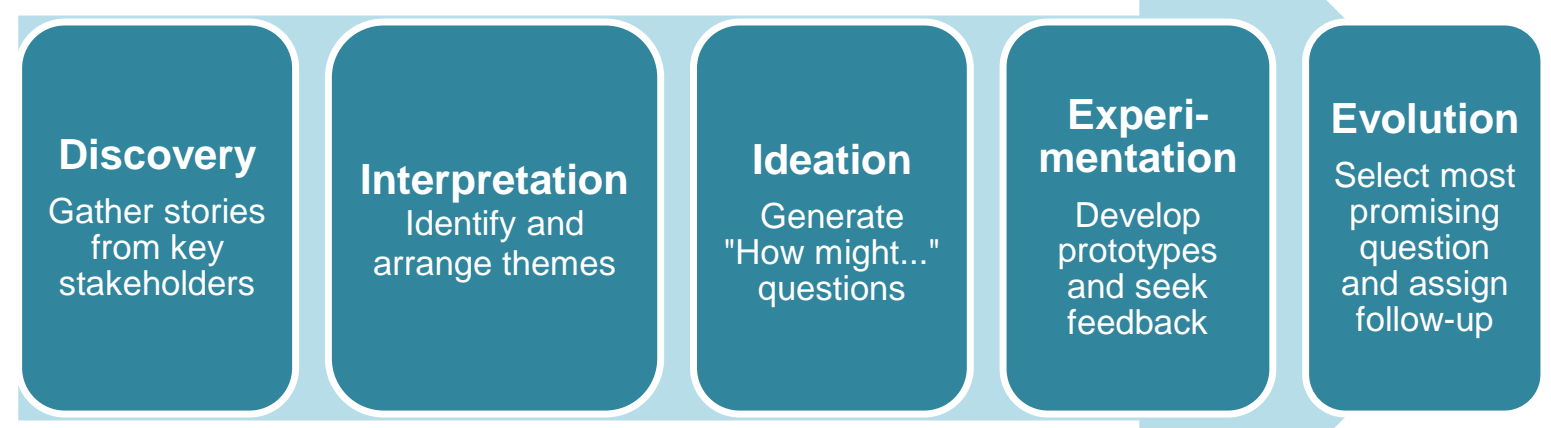

Figure 1: The five stages of design thinking as applied to the workshop.

The workshop began with a discovery phase on the morning of the first day in which participants heard perspectives of key IPE stakeholders and an overview of educational 
research from a social psychologist. To gather first-person stories, participants divided into small groups and rotated through stations where they engaged with prelicensure students in nursing, physical therapy, and speech language pathology, a recent medical school graduate, and a physician turned patient advocate.

In the interpretation phase, seminar participants worked in small groups to distill key ideas from their conversations, and then they arranged their ideas in clusters according to emerging themes. The first day ended with the ideation phase in which a facilitator helped frame the most promising themes into questions for further investigation:

- How might IPE influence the culture of the clinical site?

- How might faculty development improve IPE?

- How might the benefit of IPE justify the cost?

- How might IPE advance the Triple Aim (improved population health, improved patient experience, and lower cost)?

Day two began with the experimentation phase, where pairs or trios of participants selected one of the four questions about IPE outcomes and developed it into a fullfledged evaluation model. Testing these prototypes took the form of each group presenting ideas for evaluation research to a panel of educational researchers and receiving feedback. The group considered the merits and deficiencies of each model in the final, evolution phase to determine the most viable possibilities and chart steps for future implementation. 


\section{Results}

By the final stage, seminar participants identified the theme of organizational culture change as the most promising model for evaluating IPE because it provides an intermediate step between learning interventions and health outcomes. We conceptualized two interconnected processes: the way that IPE learners influence the culture of collaboration in a clinical setting and the way that an organization's culture of collaboration contributes to health outcomes and cost. Because classroom and clinical settings overlap so extensively in health professions education, it is also reasonable to ask whether student learning outcomes related to IPE change based on the culture of the environment in which they are precepted (Figure 2).
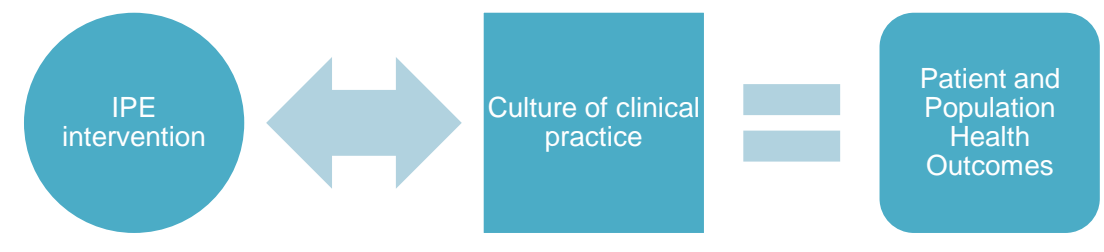

Figure 2: Conceptual model illustrating how interprofessional education and the culture of teamwork in clinical practice are mutually enforcing, contributing to health outcomes

Workshop participants, following Schein (1987), conceived of organizational culture as learned, shared understandings among a group of people that govern beliefs, behavior, and power relations. It manifests in both visible artifacts and underlying assumptions. We assumed that each setting carries its own culture, sending signals about the 
accepted way to deliver care that could either foster interprofessional collaboration or hinder it.

To test this hypothesis will require a mixed methods approach. Quantitative instruments of organizational culture that measure team relationships could be combined with a review of artifacts, ethnographic observation, and social network analysis to establish how central interprofessional collaborative practice is to an organization's culture. Because culture is dynamic and context-dependent, the goal of any evaluation project would not be to produce generalizable rules, but to identify promising attributes of practices that could be brought to scale.

\section{Discussion}

By generating and testing ideas through the design thinking process, participants arrived at a conclusion that questioned the seminar's premise. Initial efforts to justify IPE by tying it directly to patient outcomes seemed to treat IPE as an end goal in itself. However, the intended consequence of effective IPE should be enhanced collaborative practice. As Cook and West (2013) argue, establishing a causal link between medical education and patient outcomes is fraught with confounders. Isolating the health impact of interprofessional education is even more difficult. Perhaps the alternative of measuring learners' ability to carry interprofessional competencies into the work setting and the effect on team performance when such training occurs is a more reliable indicator of IPE impact. 
Seminar participants intend to continue their work refining a research agenda for the evaluation of interprofessional education. Building from a conceptual model theorizing the impact of IPE across the learning continuum, future studies can focus on key transmission points. One critical intermediate step in preparing learners to positively influence the practice environment is comprehensive faculty development (Shrader, Mauldin, Hammad, Mitcham, \& Blue, 2015). Further down the continuum, collecting both qualitative and quantitative data can help identify the key factors that characterize a "culture of collaboration." Ultimately, the aim is to synthesize research linking IPE and collaborative practice with studies showing the impact of collaborative practice on patient outcomes.

\section{Acknowledgments}

We would like to thank our interlocutors at the seminar who sparked our thinking: George E. Thibault, MD, Edward M. Hundert, MD, Samuel Moulton, PhD and colleagues at the Harvard Initiative for Learning and Teaching, Liane Fernyhough, Heather Friedman, Andrew Milinazzo, Ravi Parikh, MD, and Charlotte Yeh, MD.

\section{Declaration of interest}

The authors report no conflicts of interest. The authors alone are responsible for the writing and content of this article.

\section{Funding}

PSC received financial support from the Radcliffe Institute of Advanced Study at Harvard University to hold this workshop.

\section{References}

Brandt, B., Lutfiyya, M. N., King, J. A., \& Chioreso, C. (2014). A scoping review of interprofessional collaborative practice and education using the lens of the Triple Aim. Journal of Interprofessional Care, 28(5), 393-399.

Brown, T. (2008). Design thinking. Harvard Business Review, 86(6), 84-92. 
Cook, D. A., \& West, C. P. (2013). Perspective: Reconsidering the focus on "outcomes research" in medical education: A cautionary note. Academic Medicine, 88(2), 162-167.

Institute of Medicine. (2015). Measuring the impact of interprofessional education on collaborative practice and patient outcomes. Washington, DC: The National Academies Press.

Parker, K., Burrows, G., Nash, H., \& Rosenblum, N. D. (2011). Going beyond Kirkpatrick in evaluating a clinician scientist program: It's not "if it works" but "how it works." Academic Medicine, 86(11), 1389-1396.

Schein, E. H. (1987). Defining organizational culture. In J. M.Shafritz \& J. S.Ott (Eds.), Classics of Organizational Theory (2nd ed., pp. 381-396). Chicago, IL: The Dorsey Press.

Shrader, S., Mauldin, M., Hammad, S., Mitcham, M, \& Blue, A. (2015). Developing a comprehensive faculty development program to promote interprofessional education, practice and research at a free-standing academic health science center. Journal of Interprofessional Care, 29(2), 165-167.

Zuber, C.D., Alterescu, V., \& Chow, M. (2005). Fail often to succeed sooner: Adventures in Innovation. The Permanente Journal, 9(4), 44-49. 